\title{
Histopathological characteristics of a novel knock-in mouse prostate cancer model
}

\section{G. $\mathrm{Wu}^{1,2^{*}}, \mathrm{D}$. Wang ${ }^{1 *}$, \\ H. Wang ${ }^{1}$, J. Yuan ${ }^{1}$ and J.W. Xuan ${ }^{2}$}

\author{
${ }^{1}$ Department of Urology, Xijing Hospital, the Fourth Military Medical University, \\ Xi'an, China \\ ${ }^{2}$ Department of Surgery, University of Western Ontario, London, Ontario, Canada
}

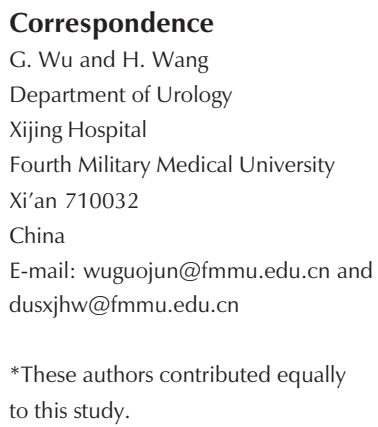

Received July 11, 2005 Accepted November 23, 2005

\begin{abstract}
Prostate cancer is relatively unique to man. There is no naturally occurring prostate cancer in the mouse. Pre-clinical studies involve the establishment of a genetically engineered mouse prostate cancer model with features close to those of the human situation. A new knock-in mouse adenocarcinoma prostate (KIMAP) model was established, which showed close-to-human kinetics of tumor development. In order to determine if the similar kinetics is associated with heterogeneous tumor architecture similar to the human situation, we utilized a new mouse histological grading system (Gleason analogous grading system) similar to the Gleason human grading system and flow cytometry DNA analysis to measure and compare the adenocarcinoma of the KIMAP model with human prostate cancer. Sixty KIMAP prostate cancer samples from 60 mice were measured and compared with human prostate cancer. Flow cytometry DNA analysis was performed on malignant prostate tissues obtained from KIMAP models. Mice with prostate cancer from KIMAP models showed a 53.3\% compound histological score rate, which was close to the human clinical average (50\%) and showed a significant correlation with age $(\mathrm{P}=0.001)$. Flow cytometry analyses demonstrated that most KIMAP tumor tissues were diploid, analogous to the human situation. The similarities of the KIMAP mouse model with tumors of the human prostate suggest the use of this experimental model to complement studies of human prostate cancer.
\end{abstract}

\section{Introduction}

Prostate cancer $(\mathrm{CaP})$, the most common cancer in adults in North America, is relatively unique to man, with no spontaneously arising $\mathrm{CaP}$ being observed in rodents in nature. It has been difficult to rapidly develop effective treatment strategies in part because there are so few animal models that accurately mimic the specific heterogeneity
Key words

- Prostate cancer model - KIMAP mouse model - Gleason grading system - Prostate adenocarcinoma 
interactions between the various cellular compartments of the prostate gland. For this reason, the development of genetically engineered mouse $\mathrm{CaP}$ models is critical for preclinical studies of $\mathrm{CaP}(1-10)$.

Prostate secretory protein of 94 amino acids (PSP94), also known as B-microseminoprotein, like human prostate-specific antigen (PSA), is one of the most abundantly expressed secretory proteins in human prostatic fluid and semen $(11,12)$. Elevated serum levels of PSA and PSP94 have been identified and used as serum markers for the diagnosis and prognosis of $\mathrm{CaP}$. In contrast to PSA, which has no equivalent in rodents, PSP94 from humans, primates, pigs, and rodents is conserved, but is also a rapidly evolving protein (13-16).

We have established a new knock-in mouse adenocarcinoma of the prostate (KIMAP) model line at the PSP94 gene locus $(17,18)$. The new KIMAP model proved to have several advantages over the traditional transgenic models, such as prolonged tumor growth, a predominance of well- and moderately differentiated tumors, highly synchronous prostate cancer development, and highly stable phenotype and genotype (18). In the present study, we utilized a new mouse histological grading system (Gleason analogous grading system) similar to the Gleason human grading system and flow cytometry DNA analysis to measure and compare prostate tissue of the new KIMAP model with human $\mathrm{CaP}$ tissue. The present study demonstrates the similarity of this new murine prostate cancer model with human $\mathrm{CaP}$ and suggests that KIMAP could be used as an experimental model together with the transgenic models.

\section{Material and Methods}

\section{Histological characterization and definition of various grades of prostate cancer in knock-in mice}

To study tumor development in the ge- netically engineered PSP-knock-in (KIMAP) model, some modifications were adopted according to the established diagnostic criteria previously reported (19-21). In view of the heterogeneity of the clinical standard for $\mathrm{CaP}$ diagnosis, a close-to-human mouse standard for histological grading and scoring system was established in the present study. We termed this system the "Gleason analogous grading system". The architectural patterns of adenocarcinoma observed were scored according to 5 different histological grades: grade 1 (very well-differentiated): single, separate, uniform glands closely packed, with definite boundaries; grade 2 (well-differentiated): single, separate uniform glands loosely packed, with irregular edges; grade 3 (glands with variable and distorted architecture): single, separate, uniform scattered glands, and smoothly circumscribed papillary/cribriform masses; grade 4 (poorly differentiated): cribriform masses with ragged, invading edges and fused glands; grade 5: non-glandular solid, rounded masses of cells, cribriform architecture with foci of central necrosis (known as comedocarcinoma) and undifferentiated anaplastic carcinomas. Based on the most prevalent histological grade ("the primary pattern/ grade") and the second most prevalent histological pattern ("secondary pattern/grade"), the new mouse scoring system was derived by adding the primary pattern grade number to the secondary grade number. If only one pattern is seen throughout, the score is derived by doubling the "grade" number.

\section{Anatomy, identification and breeding of knock-in mice by PCR genotyping}

All animal experiments were conducted according to standard protocols. All KIMAP mouse breeding lines were established mostly in the CD1 or $129 \mathrm{~Sv}$ background, and the C57BL6 background was also tested with no strain differences. Genotyping was performed by fast PCR genotyping as previ- 
ously reported (18). The primer pairs used for screening germline progenies from chimeras by PCR genotyping were as previously reported $(17,18)$.

The prostate along with the male accessory glands, i.e., the ventral and dorsolateral prostate lobes, seminal vesicles and coagulation gland (or the anterior gland), were dissected out separately as previously described and defined $(15,17)$.

\section{Flow cytometry}

Flow cytometry DNA analysis was performed on malignant prostate tissues obtained from the KIMAP model $(\mathrm{N}=9)$, which were either freshly excised or snap frozen in liquid nitrogen. Single cell suspensions were prepared according to the standard clinical procedures of our hospital. Prior to analysis, each cell suspension was filtered through a nylon mesh to remove any debris and cell aggregates. Normal mouse spleen lymphocytes were dissociated and used as a control to establish the normal diploid DNA peak position. All samples were analyzed with an EPICS C flow cytometer (Coulter Electronics, Hialeah, FL, USA). The resultant single nucleus suspension was treated with ribonuclease and stained with $50 \mathrm{~g} / \mathrm{mL}$ propidium iodide (Beckman Coulter, Inc., Miami, FL, USA). DNA histograms were classified as diploid, tetraploid, or aneuploid. DNA aneuploidy was defined by the presence of a tumor population with a definable G0/G1 peak which was distinct from the diploid population. Tetraploidy was defined by a peak with a DNA index (ratio of the channel number of the abnormal to the diploid population) of 1.9 to 2.1. DNA proliferation was measured by flow cytometry as values of $\% \mathrm{~S}$ (percent $\mathrm{S}$ phase) and $\% \mathrm{~S}+\% \mathrm{G}_{2} \mathrm{M}$ (percent $\mathrm{S}$ phase and $\mathrm{G}_{2} \mathrm{M}$ phase) separately (22).

\section{Statistical analysis}

The SPSS 10 and Sigma Plot 2000 softwares were used for all analyses, including the $\chi^{2}$ test and Mann-Whitney test.

\section{Results}

\section{Comparison of KIMAP mouse prostate}

tumors with human prostate tumors by the

Gleason analogous grading system

The gross pathology of a KIMAP mouse 52 weeks of age with prolonged $\mathrm{CaP}$ development is shown in Figure 1A. In order to differentiate the heterogeneity of the architecture of mouse prostate tumor, we established a new mouse histological grading system similar to the human Gleason grading system. Each KIMAP mouse with $\mathrm{CaP}(\mathrm{N}=$ 60 ) was assigned a combination of a primary histological grade (a dominant grade) and a secondary grade (the non-dominant grade) using the mouse Gleason analogous grading

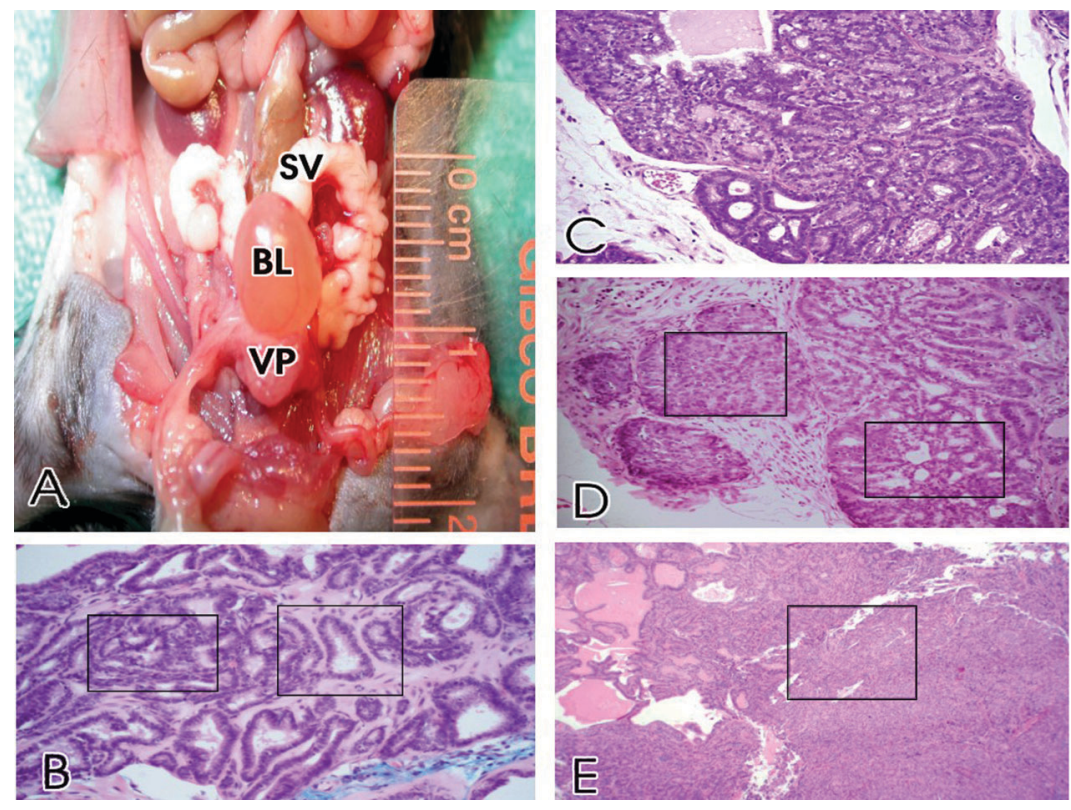

Figure 1. Gross pathology and histological analysis of the knock-in mouse adenocarcinoma prostate (KIMAP) model by a new mouse Gleason analogous grading system. A, Gross pathology of a KIMAP mouse 52 weeks of age after prolonged prostate cancer development showing a 0.3-g ventral prostate lobe tumor. $B$, Histological score 5 from a KIMAP mouse at age 30 weeks. Two boxes show histological grade 2 (right) and 3 (left) tumor foci. 25X. $C$, Histological score 6 (histological grades $3+3$ ) for a KIMAP mouse at age 30 weeks. 25X. $D$, Histological score $7(3+4)$ for a KIMAP mouse at age 45 weeks. Right area, grade 3 , small acinar infiltrating the stroma. Left area, grade 4 showing cribriform acini. 10X. E, Histological score $9(4+5)$ for a KIMAP mouse at age 67 weeks. Boxed area, grade 5 . Left area, grade 4. 10X. All sections were stained with hematoxylin and eosin. $S V=$ seminal vesicle; $B L=$ bladder; VP = ventral prostate lobe tumor. 
Figure 2. Histological scores (compound score percentage) for knock-in mouse adenocarcinoma prostate model (KIMAP) mice. $A$, Distribution of the histological scores of the KIMAP model. The numbers above each bar in the graph indicate the number of mice, with the histological scores indicated on the abscissa. Percentage (ordinate) indicates the percentage of mice with the identified histological scores among the total mice tested. $B$, Plot of the compound histological score percentages (\% histological scores composed of different primary and secondary scores) against age groups. The numbers above each bar indicate the number of mice of that age group with the identified compound score rate (\%). system (see boxes in Figure 1B,D,E). The distribution of KIMAP histological scores is plotted in Figure 2A. Mouse histological scores were evenly distributed in all 4-9 ranges except the score 10 (doubling grades $5+5)$. Most scores were $9(28.3 \%)$ and 6 (23.3\%), and other scores were 4 (5.0\%), 5 (5.0\%), $7(20.0 \%)$, and $8(18.3 \%)$. The histological score distribution for KIMAP was close to reported clinical data, i.e., 4(14.3\%), 5 (15.3\%), 6 (42.2\%), 7 (19.1\%), and $\geq 8$ (9.2\%) $(20,21)$.

\section{KIMAP animals showed a closer-to-human compound histological score rate}

The architectural heterogeneity of tumors in the KIMAP model was further characterized by the percentage of the compound histological score rate, which was designated as the proportion (\%) of mice with the compound histological scores (combination of two unequal histological grades) among

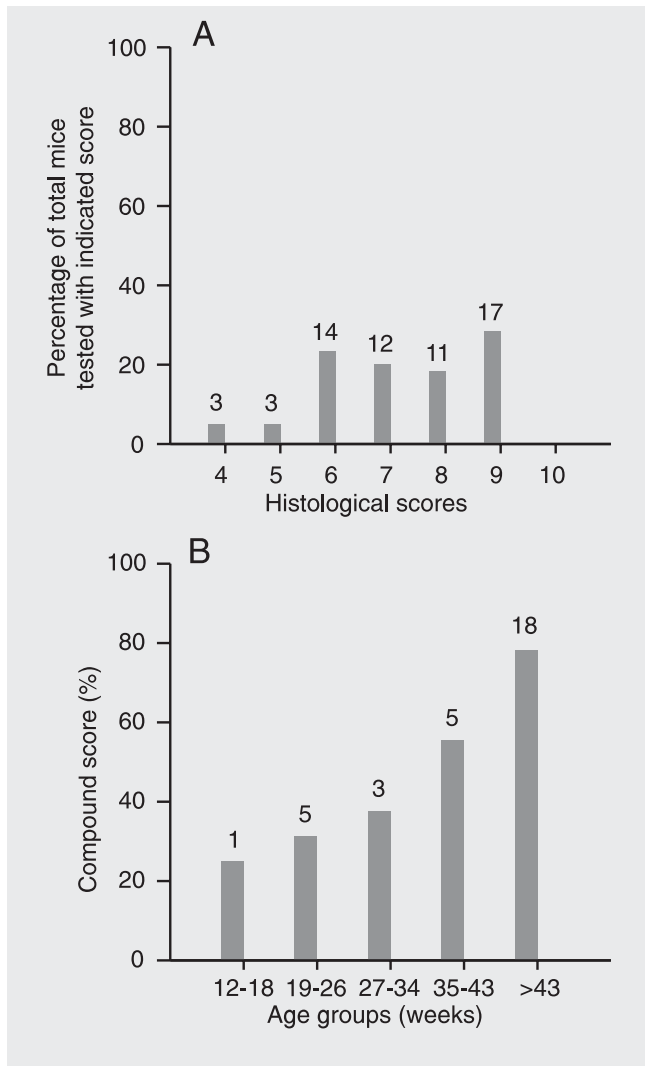

the total $\mathrm{CaP}$ mice (shown in boxes in Figure $1)$. The average $\%$ compound histological score rates in KIMAP was $53.3 \%(\mathrm{~N}=60)$, which is very close to the reported clinical average of $50 \%(20,21)$. Figure 1 shows the assessment of compound mouse histological score 5 (2+3, Figure 1B), 7 (3+4, Figure 1D) and $9(4+5$, Figure 1E) in KIMAP mice.

Next, we determined the correlation of $\%$ compound score rate with age, as well as the starting age for this process resulting in the different $\%$ compound histological score rates. The distribution of the $\%$ compound histological score rates (Figure 2B) was plotted against different age groups. The majority of KIMAP mice (32/60) showed high \% compound scores, distributed among age groups (from 12 weeks of age) as follows: $25 \%$ (12-18 weeks), 31.3\% (19-26 weeks), $37.5 \%$ (27-34 weeks), 55.6\% (35-43 weeks), and $78.3 \%$ ( $>43$ weeks) (Figure 2B). KIMAP animals showed a trend to an increased compound score rate which correlated with age $(\mathrm{P}=0.001$, Figure 2B).

\section{Flow cytometry studies}

In order to further characterize the murine model at the chromosomal level, flow cytometry experiments were performed. Homogenous prostate tumors were collected only from late stage mice. Diploid control tissue samples were obtained from mouse normal spleen tissue cells (Figure 3A). In the 9 KIMAP tumor samples analyzed, 8 mice showed mostly diploid tumor peaks (89\%, Figure $3 \mathrm{~B}$ and $\mathrm{C}$ ) and only one mouse revealed a tetraploid peak (11\%, Figure 3D), which approximate the results of flow cytometry in clinical $\mathrm{CaP}$ investigation $(75 \%$ diploid and 25\% non-diploid tumor (22)). Figure $3 \mathrm{~B}$ and $\mathrm{C}$ shows the results for the sample plus spleen test and for the sample alone (without the spleen control) separately.

The DNA proliferation rate $(\% \mathrm{~S}$ and $\% \mathrm{~S}$ $\left.+\% \mathrm{G}_{2} \mathrm{M}\right)$ of KIMAP was assessed by flow 
cytometry. The average $\% \mathrm{~S}$ and $\% \mathrm{~S}+\% \mathrm{G}_{2} \mathrm{M}$ of KIMAP animals were 3.6 and $10.7 \%$, respectively, closely reflecting the DNA proliferation rate reported in clinical studies (3.1 and $10.5 \%$, respectively) (22).

\section{Discussion}

Due to the biologic heterogeneity of $\mathrm{CaP}$, further understanding of the biology of $\mathrm{CaP}$ is necessary before significant advances in therapy can be obtained. Insight into the epigenetic events and cellular interactions between $\mathrm{CaP}$ cells and the organ microenvironment will be critical. Continued empirical treatment is unlikely to provide the therapeutic advances required to improve outcomes for patients with $\mathrm{CaP}$. The use of animal models may provide an experimental or in vivo system to study $\mathrm{CaP}$ biology. $\mathrm{CaP}$ continues to be the second leading cause of cancer-related death among men. To develop more fully effective prevention and intervention strategies for this prevalent disease, the underlying molecular mechanisms
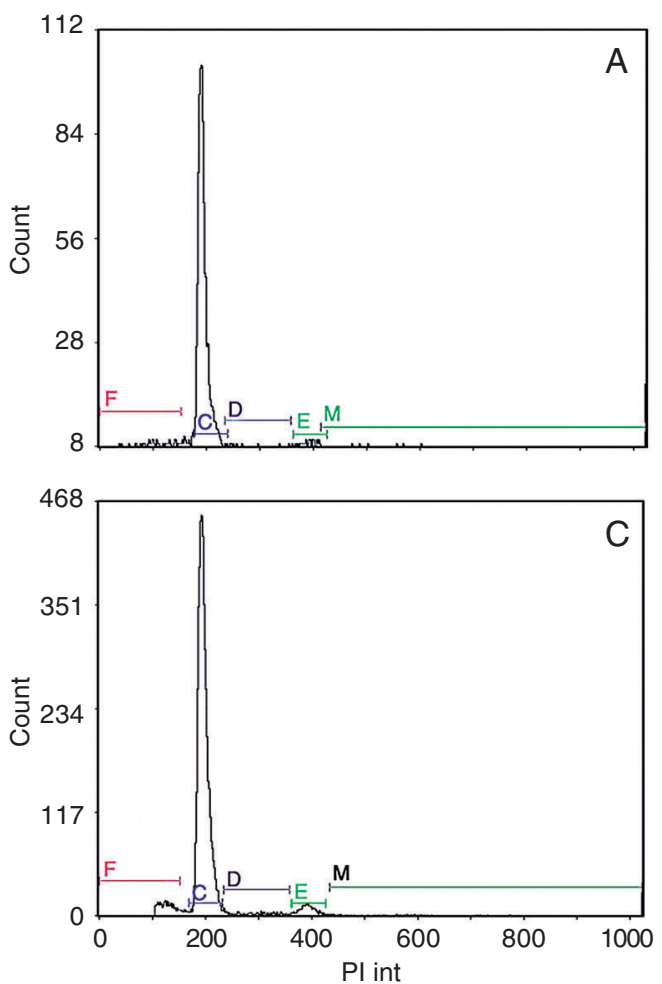

of initiation, progression, and metastatic spread must be understood. To this end, mouse models can contribute significantly to $\mathrm{CaP}$ research in that they can mimic the pathological and biochemical features of the disease.

A mouse model mimicking human $\mathrm{CaP}$ must have the following features which are characteristic of human $\mathrm{CaP}$ : correlation with increasing age, high incidence rate, slow growth rate, a histological $\mathrm{CaP}$ structure mainly involving well to moderate differentiation, androgen dependence, and initial responsiveness to hormone therapy followed by the development of refractoriness to androgen ablation therapy, and high propensity for bone metastasis.

The Gleason grading system is based on the degrees of architectural differentiation and has been advocated as a way to improve the pathologists' ability to make an accurate diagnosis and prognosis of the biological behavior of a particular tumor. This system effectively detects the full spectrum of human $\mathrm{CaP}$ development. Other internation-
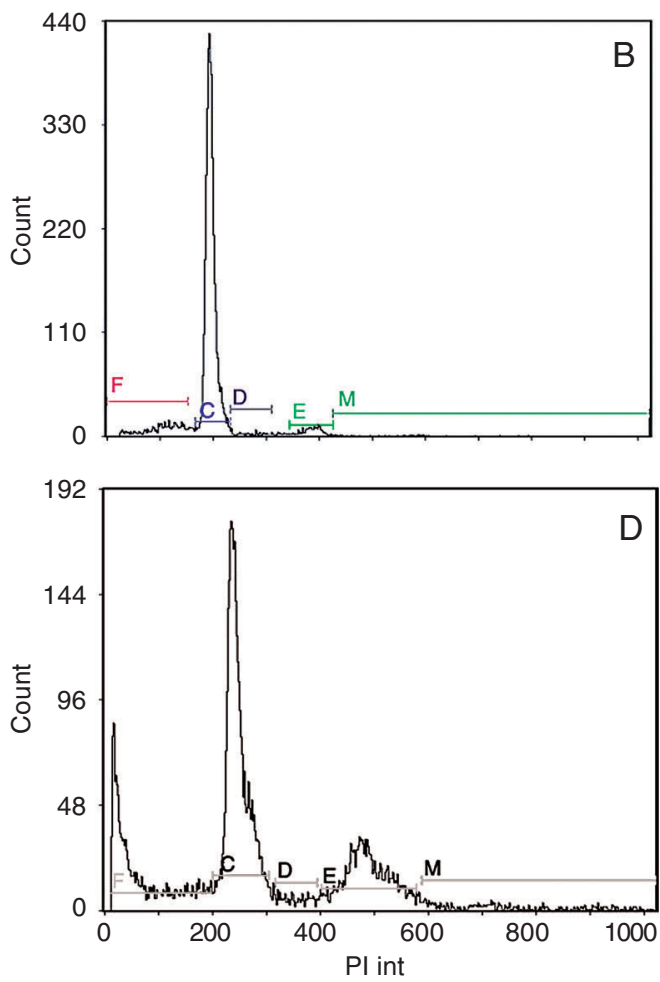

Figure 3. Flow cytometry analysis of late stage prostate cancer $(\mathrm{CaP})$ samples from the knockin mouse adenocarcinoma prostate (KIMAP) model. All diagrams are singlet pictures, with cell counts (ordinate) plotted against $\mathrm{PI}$ int (propidium iodide $p$-iodonitrotetrazolium). $A$, Normal spleen tissue dissociated cells from KIMAP mice (control for diploid); $B$, mixture of spleen and tumor cells of KIMAP mice showing diploidy; $C$, test of $\mathrm{CaP}$ cells alone from KIMAP mice showing diploidy; $D$, test of $\mathrm{CaP}$ cells alone from KIMAP mice showing tetraploid changes (peak E). Peaks correspond to $C$ (cell cycle phase G0/G1, for diploidy), D (S phase for aneuploidy), and $E\left(G_{2} M\right.$ phase for tetraploidy). 
ally used systems are the Mostofi (World Health Organization) and Boking systems. These grading systems can identify welldifferentiated and poorly differentiated adenocarcinomas, but are less successful in subdividing moderately differentiated adenocarcinomas.

The KIMAP model is a mouse model resulting from a single endogenous knockin mutation under the control of the promoter/enhancer region of the prostate-specific gene PSP94. One of the unique features of this PSP-KIMAP model is the presence of sufficient heterogeneity to permit the use of a system similar to the human Gleason histological grading system, which is extensively used in clinical practice for the grading of human prostate cancer $(18,20,21)$. We have named this system, created for our study purposes, the "Gleason analogous grading system". The most prevalent range of Gleason analogous grades (3-4) and Gleason analogous scores (5-7/10) was the same in PSPKIMAP mice and in human CaP cases (21). Additionally, a significant correlation of Gleason analogous grades and scores with animal age was observed. KIMAP mice also demonstrated a high percentage of compound histological scores, a feature that is more analogous to human CaP. Flow cytometry study of DNA ploidy and proliferation demonstrated that the majority of KIMAP mouse tumor cells were diploid, as in human $\mathrm{CaP}$ (22).

Mouse $\mathrm{CaP}$ models cannot be applied to preclinical studies if they do not show all of the histopathologic patterns of CaP. None of the previous transgenic $\mathrm{CaP}$ models have demonstrated features that would qualify them for classification by the Gleason analogous grading system. However, all previous transgenic models can only be characterized by a "crude," non-clinically relevant histological grading system (23), partly due to the lack of sufficient heterogeneity and rare patterns of neoplastic change.

The PSP-KIMAP model revealed a similarity to the human situation in its histopathological characteristics. The simplest theoretical explanation for this is that none of the previously described transgenic $\mathrm{CaP}$ models is fully regulated because of an endogenous knock-in mutation in a prostate-specific gene. The knocked insertion of the PSP94 gene confers high regularity upon the PSP-KIMAP model since it only knocks in an SV40 Tag in the PSP94 structural gene, without affecting any regulatory factors/regions. The PSP94 gene promoter is a strong, prostate tissue-specific promoter, and the SV40 Tag expression is controlled by it.

As with all genetically engineered prostate cancer models, a mouse-specific histopathological standard cannot be directly applied to human clinical diagnosis or prognosis. However, the PSP-KIMAP model, which most closely mimics human $\mathrm{CaP}$, may reveal more similarities of tumor development to $\mathrm{CaP}$ and provide an experimental approach to the study of prostate tumors.

\section{References}

1. Abate-Shen C, Shen MM. Mouse models of prostate carcinogenesis. Trends Genet 2002; 18: S1-S5.

2. Huss WJ, Maddison LA, Greenberg NM. Autochthonous mouse models for prostate cancer: past, present and future. Semin Cancer Biol 2001; 11: 245-260.

3. Winter SF, Cooper AB, Greenberg NM. Models of metastatic prostate cancer: a transgenic perspective. Prostate Cancer Prostatic Dis 2003; 6: 204-211.

4. Green JE, Greenberg NM, Ashendel CL, Barrett JC, Boone C, Getzenberg $\mathrm{RH}$, et al. Workgroup 3: transgenic and reconstitution models of prostate cancer. Prostate 1998; 36: 59-63.

5. Killion JJ, Radinsky R, Fidler IJ. Orthotopic models are necessary to predict therapy of transplantable tumors in mice. Cancer Metastasis Rev 1998; 17: 279-284.

6. Masumori N, Thomas TZ, Chaurand P, Case T, Paul M, Kasper S, et al. A probasin-large $T$ antigen transgenic mouse line develops prostate adenocarcinoma and neuroendocrine carcinoma with metastatic potential. Cancer Res 2001; 61: 2239-2249.

7. Maroulakou IG, Anver M, Garrett L, Green JE. Prostate and mammary adenocarcinoma in transgenic mice carrying a rat $\mathrm{C} 3(1)$ sim- 
ian virus 40 large tumor antigen fusion gene. Proc Natl Acad Sci USA 1994; 91: 11236-11240.

8. Garabedian EM, Humphrey PA, Gordon JI. A transgenic mouse model of metastatic prostate cancer originating from neuroendocrine cells. Proc Natl Acad Sci USA 1998; 95: 15382-15387.

9. Perez-Stable C, Altman NH, Mehta PP, Deftos LJ, Roos BA. Prostate cancer progression, metastasis, and gene expression in transgenic mice. Cancer Res 1997; 57: 900-906.

10. Ellwood-Yen K, Graeber TG, Wongvipat J, Iruela-Arispe ML, Zhang $\mathrm{J}$, Matusik R, et al. Myc-driven murine prostate cancer shares molecular features with human prostate tumors. Cancer Cell 2003; 4: 223-238.

11. Hara M, Kimura H. Two prostate-specific antigens, gammaseminoprotein and beta-microseminoprotein. J Lab Clin Med 1989; 113: 541-548.

12. Abrahamsson PA, Lilja H, Falkmer S, Wadstrom LB. Immunohistochemical distribution of the three predominant secretory proteins in the parenchyma of hyperplastic and neoplastic prostate glands. Prostate 1988; 12: 39-46.

13. Hyakutake $H$, Sakai $H$, Yogi $Y$, Tsuda R, Minami $Y$, Yushita $Y$, et al. Beta-microseminoprotein immunoreactivity as a new prognostic indicator of prostatic carcinoma. Prostate 1993; 22: 347-355.

14. Xuan JW, Kwong J, Chan FL, Ricci M, Imasato $\mathrm{Y}$, Sakai $\mathrm{H}$, et al. cDNA, genomic cloning, and gene expression analysis of mouse PSP94 (prostate secretory protein of 94 amino acids). DNA Cell Biol 1999; 18: 11-26.

15. Imasato $\mathrm{Y}$, Onita $\mathrm{T}$, Moussa M, Sakai H, Chan FL, Koropatnick J, et al. Rodent PSP94 gene expression is more specific to the dorsolateral prostate and less sensitive to androgen ablation than probasin. Endocrinology 2001; 142: 2138-2146.
16. Lazure C, Villemure M, Gauthier D, Naude RJ, Mbikay M. Characterization of ostrich (Struthio camelus) beta-microseminoprotein (MSP): identification of homologous sequences in EST databases and analysis of their evolution during speciation. Protein Sci 2001; 10: $2207-2218$.

17. Gabril MY, Onita T, Ji PG, Sakai H, Chan FL, Koropatnick J, et al. Prostate targeting: PSP94 gene promoter/enhancer region directed prostate tissue-specific expression in a transgenic mouse prostate cancer model. Gene Ther 2002; 9: 1589-1599.

18. Duan W, Gabril MY, Moussa M, Chan FL, Sakai H, Fong G, et al. Knockin of SV40 Tag oncogene in a mouse adenocarcinoma of the prostate model demonstrates advantageous features over the transgenic model. Oncogene 2005; 24: 1510-1524.

19. Shappell SB, Thomas GV, Roberts RL, Herbert R, Ittmann MM, Rubin MA, et al. Prostate pathology of genetically engineered mice: definitions and classification. The consensus report from the Bar Harbor meeting of the Mouse Models of Human Cancer Consortium Prostate Pathology Committee. Cancer Res 2004; 64: 2270-2305.

20. Deshmumukh N, Foster CS. Grading prostate cancer. In: Foster CS, Bostwick DG (Editors), Pathology of the prostate cancer. Philadelphia: Saunders; 1998.

21. Bostwick DG. Neoplasms of the prostate. In: Bostwick DG, Eble JN (Editors), Urologic surgical pathology. St. Louis: Mosby; 1997.

22. So MJ, Cheville JC, Katzmann JA, Riehle DL, Lohse CM, Pankratz VS, et al. Factors that influence the measurement of prostate cancer DNA ploidy and proliferation in paraffin embedded tissue evaluated by flow cytometry. Mod Pathol 2001; 14: 906-912.

23. Greenberg NM, DeMayo F, Finegold MJ, Medina D, Tilley WD, Aspinall JO, et al. Prostate cancer in a transgenic mouse. Proc Natl Acad Sci USA 1995; 92: 3439-3443. 